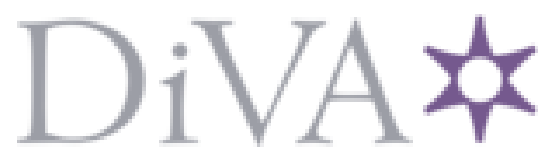

http://www.diva-portal.org

\title{
Postprint
}

This is the accepted version of a paper published in Ultrasound in Medicine and Biology. This paper has been peer-reviewed but does not include the final publisher proof-corrections or journal pagination.

Citation for the original published paper (version of record):

Widman, E., Caidahl, K., Heyde, B., D'hooge, J., Larsson, M. (2015)

Ultrasound speckle tracking strain estimation of in vivo carotid artery plaque with in vitro sonomicrometry validation.

Ultrasound in Medicine and Biology, 41(1): 77-88

http://dx.doi.org/10.1016/j.ultrasmedbio.2014.06.013

Access to the published version may require subscription.

N.B. When citing this work, cite the original published paper.

Permanent link to this version:

http://urn.kb.se/resolve?urn=urn:nbn:se:kth:diva-159741 


\section{Ultrasound Speckle Tracking Strain Estimation of In vivo Carotid Artery Plaque with In vitro Sonomicrometry Validation}

Erik Widman, ${ }^{*}$ Kenneth Caidahl, ${ }^{\dagger}$ Brecht Heyde, ${ }^{\ddagger}$ Jan D’hooge, ${ }^{\ddagger}$ and Matilda Larsson ${ }^{*}$

* Department of Medical Engineering, School of Technology and Health, KTH Royal Institute of Technology, Stockholm, Sweden

${ }^{\dagger}$ Department of Molecular Medicine and Surgery, Karolinska Institutet, Stockholm, Sweden

${ }^{\ddagger}$ Cardiovascular Imaging \& Dynamics, Department of Cardiovascular Sciences, KU Leuven, Leuven, Belgium

\section{Corresponding author}

Erik Widman

School of Technology and Health, KTH Royal Institute of Technology

Alfred Nobels Allé 10

14152 Huddinge

Sweden

Telephone number: +4687904850

Fax number: + 468218368

erik.widman@sth.kth.se 
1 Abstract- Our objective was to validate a previously developed speckle tracking (ST) algorithm to 2 assess strain in common carotid artery (CCA) plaques. Radial and longitudinal strain was measured 3 in CCA gel-phantoms with a plaque mimicking inclusion using an in-house ST algorithm and 4 sonomicrometry. Moreover, plaque strain by ST from 7 patients ( $77 \pm 6$ years) with carotid 5 atherosclerosis was compared with a quantitative visual assessment by two experienced physicians. 6 In vitro, good correlation existed between ST and sonomicrometry peak strain both radially $(\mathrm{r}=$ $7 \quad 0.96, \mathrm{p}<0.001)$ and longitudinally $(\mathrm{r}=0.75, \mathrm{p}<0.01)$. In vivo, a greater pulse-pressure adjusted 8 radial and longitudinal strain was found in echolucent plaques compared to echogenic plaques. This 9 study shows the feasibility of ultrasound ST strain estimation in plaques and indicates the 10 possibility to characterize plaques using ST strain in vivo.

11

12 Keywords - Carotid Artery; In vitro; In vivo; Phantom; Plaque Characterization; Speckle 13 Tracking; Strain; Ultrasound; Validation 


\section{INTRODUCTION}

2 Plaque characterization is critical when determining treatment for patients with carotid

3 atherosclerotic plaque (Mono et al. 2012; Reid 1998). One type of plaques, known as vulnerable

4 plaques, have a high probability of rupture causing emboli to break off from the plaque and travel

5 via the circulatory system to vessels in the brain. As the arteries become smaller the emboli can

6 lodge in the vessel wall restricting the blood flow to parts of the brain yielding a temporary transient

7 ischemic attack or permanent embolic stroke (Reckless and Buchan 2008). Plaque characterization

8 in the carotid artery is therefore significant in risk assessment for cerebral ischemic events.

9 In current clinical practice, ultrasound-based methods for plaque characterization are limited to

10 visual assessment of plaque morphology, hypoechoic area, and echo reflection in the plaque (Gray-

11 Weale et al. 1988; Kakkos et al. 2013), or more commonly in research, computerized evaluation of

12 echogenic properties (Prahl et al. 2010). Limitations of visual assessment include inter-observer

13 variability, human error, and misclassification due to ultrasound machine settings (Arnold et al.

14 1999). Other clinical practices for carotid plaque detection include carotid angiography (Armas et

15 al. 1981; Matos et al. 2014), although angiography only detects plaque location and size and does

16 not offer information about plaque composition. Treatment options, such as an endarterectomy or

17 angioplasty, are often based on relative lumen diameter. The accepted criterion for surgical

18 selection of patients with symptomatic carotid artery disease is a stenosis of $70 \%$ reduction in

19 relative lumen diameter of the internal carotid artery (Arnold et al. 1999). In certain instances of

20 plaque composition, location, and luminary occlusion; surgery poses a higher risk to the patient than

21 noninvasive treatments such as medication (Easton and Wilterdink 1994). Quantitative tools are

22 needed to better assess the likelihood of plaque rupture to determine if invasive treatment options

23 are required, particularly in cases with borderline lumen obstruction. 
1 Plaque characterization has been attempted with a number of imaging modalities and techniques.

2 Magnetic resonance imaging (MRI) has been used to characterize plaque but is expensive, time 3 consuming, and can be a high risk factor initiating recurrent plaque rupture events (Ronen et al. 4 2007; Teng et al. 2011; Zhao et al. 2013). Saba et al. (2013) attempted to use computerized 5 tomography (CT) to characterize plaque based on the Hounsfield unit (HU) values, but found that 6 the plaque HU level significantly changed according to the applied kiloelectron volt.

7 Non-invasive ultrasound-based methods include measuring the gray-scale median (GSM) of 8 plaques (Biasi et al. 1999; Kanber et al. 2013a; Salem et al. 2012), although conflicting studies 9 question the effectiveness of GSM as an indicator for increased risk of stroke (Biasi et al. 2004; 10 Froio and Biasi 2007; Reiter et al. 2006). Furthermore, GSM variability can occur between different 11 ultrasound machine manufactures or between different users depending on the ultrasound machine settings. Plaque characterization by scatter size and attenuation coefficient has been attempted, but no in vivo studies have been conducted (Shi et al. 2008b). Thermal Strain Imaging (TSI) has been 14 used in vivo to detect lipids in atherosclerotic plaques in the femoral arteries of rabbits (Mahmoud et al. 2013). Detection of vulnerable carotid plaque has also been performed using contrast-enhanced ultrasound imaging in combination with Tissue Doppler imaging (Deyama et al. 2013), but the 17 usefulness of Tissue Doppler imaging has been found limited due to high variability (Ramnarine et 18 al. 2003).

Two new ultrasound-based elastography methods that have been used to characterize plaque are Acoustic Radiation Force Impulse imaging (ARFI) (Allen et al. 2011; Dahl et al. 2009) and Shear

21 Wave Elastography (SWE) (Ramnarine et al. 2014; Widman et al. 2012). ARFI imaging can readily 22 distinguish between stiff and soft tissue regions, but can only detect relative stiffness differences in 23 the plaque to the nearby surrounding tissue. SWE still needs to be adapted for plaque measurements 
1 since current results have neglected the viscoelasticity of the carotid artery as well as the shear wave

2 phase aberration effects due to the cylindrical geometry of the vessel.

3 Atherosclerotic plaque strain is another measure that has been suggested for detection of plaques

4 prone to rupture. Ultrasound-based speckle tracking is a block-matching technique that estimates

5 strain by tracking interference patterns across imaging frames. Ultrasound speckle tracking plaque

6 characterization has been shown feasible with intravascular ultrasound (IVUS) (de Korte et al.

7 2002; Le Floc'h et al. 2012; Schaar et al. 2003). Moreover, attempts to non-invasively assess plaque

8 strain by radiofrequency based ultrasound speckle tracking have been performed (McCormick et al.

9 2012; Schmitt et al. 2007).

10 The driving forces of arterial strain are thought to vary with different types of strain. Radial and

11 circumferential strains are caused from the blood pressure wave during the cardiac cycle. Given

12 conservation of wall volume, radial strain will have a waveform inverse to the pressure wave and be

13 of similar shape. Longitudinal strain is more complex and thought to be caused by a combination of

14 the pressure wave, wall shear stress from the blood, and tethering of the vessel due to the 15 contracting heart.

16 Even though several plaque characterization techniques have been proposed, noninvasive 17 techniques with extensive validation via independent reference methods are still missing to the best 18 of our knowledge. Of the clinically available imaging modalities it is advantageous to use B-mode 19 ultrasound to characterize plaque due to its low cost, accessibility, and safety. Recently, our 20 research group published a speckle tracking algorithm based on block matching that successfully 21 estimated strain in the wall of a carotid artery in silico (Larsson et al. 2011a), in vitro (Larsson et al. 22 2011b), and validated in vivo in a sheep model (Larsson et al. 2013). The aim of this study was to 23 use this speckle tracking algorithm to validate radial and longitudinal strain assessment in plaques 24 by comparing the speckle tracking measurements against sonomicrometry in tissue-mimicking 
1 phantoms. Additionally, this study attempted to preliminary demonstrate the feasibility of in vivo

2 plaque strain measurements in patients with atherosclerotic plaques, with the hypothesis that soft

3 vulnerable plaques with a large lipid or intraplaque hemorrhage core should exhibit larger strain

4 compared to more stable fibrous plaques.

5 METHODS

6 The in-house strain estimation algorithm was first tested in a phantom setup mimicking the 7 carotid artery and validated via sonomicrometry to ensure the accuracy of the algorithm. Upon 8 validation, in vivo ultrasound cineloops were collected in patients with carotid atherosclerotic 9 plaques in which the strain was calculated.

\section{IN VITRO EXPERIMENTS}

\section{A. Vessel Phantom Construction}

Four in vitro carotid artery phantoms with a plaque mimicking inclusion were constructed from a mixture (w/w) of $87 \%$ deionized water, 10\% polyvinyl alcohol (PVA) with a molecular weight of $1456.140 \mathrm{~g} / \mathrm{mol}$ (Sigma-Aldrich, St. Louis, MO, USA) and 3\% graphite powder with particle size < $50 \mu \mathrm{m}$ (Merck KGaA, Darmstadt, Germany). The solution was heated and stirred until the mixture thickened and was fully dissolved. It was subsequently poured in the vessel phantom mold (Fig. 1a),

17 which consisted of a hollow acrylic block (cylindrical shape with a $12 \mathrm{~mm}$ diameter, $100 \mathrm{~mm}$ 18 length, and molded fixing collars) and a bronze rod with a $6 \mathrm{~mm}$ diameter placed in the center of the

19 hole. The metallic rod had a $50 \mathrm{~mm}$ long and $1.5 \mathrm{~mm}$ thick extension attached to create a cavity in 20 the phantom wall (Fig. 1b).

21 Once the solution was poured in the mold, it stayed confined between the acrylic block and the 22 rod, resulting in a tube-shaped phantom. The phantom was frozen for 12 hours at 23 approximately $-23{ }^{\circ} \mathrm{C}$ and then thawed for 12 hours at room temperature $\left(\approx 20{ }^{0} \mathrm{C}\right)$. This freeze- 
1 thaw cycle was repeated twice for three phantoms and three times for one of the phantoms.

2 Subsequently, the phantom wall cavity was filled with a PVA-graphite solution, prepared the same

3 way as described above, and the entire phantom was exposed to one extra freeze-thaw cycle,

4 resulting in three and four freeze-thaw cycles for the vessel wall and one for the cavity mimicking

5 the plaque. Since PVA stiffens with increasing freeze-thaw cycles, the phantom wall became stiffer

6 than the inclusion, mimicking the vessel wall and the soft plaque, respectively. A photo of the

7 acrylic mold and rod as well as a schematic representation of the vessel phantom is shown in Fig. 1.

8 As the plaque cavity was filled with PVA, the thin vessel wall $(0.75 \mathrm{~mm})$ extruded into the lumen

9 creating a partial lumen occlusion shown in Fig. 2 b.

\section{B. Experimental Setup}

11 The PVA-phantoms were attached to a polyvinyl chloride (PVC) box (100 $\mathrm{mm} \times 85 \mathrm{~mm} \times$

$12300 \mathrm{~mm}$ ) by placing two plastic discs around the fixing collars of the phantoms and tightening them

13 to the fixture with screws (Fig. 2a). The phantoms were aligned in the fixture with the plaque

14 positioned in the posterior wall. In order to simulate the tissue surrounding the carotid artery a

15 mixture (w/w) consisting of 3\% agar (Merck KGaA, Darmstadt, Germany), 4\% graphite power, and

$1693 \%$ deionized water by mass was heated while continuously stirred to $73{ }^{\circ} \mathrm{C}$, then cooled to $40{ }^{\circ} \mathrm{C}$,

17 and subsequently poured into the PVC-box until the PVA-vessel phantom was covered with 18 approximately $1 \mathrm{~cm}$ of agar-mixture.

19 The phantoms were connected to a programmable pump (CompuFlow 1000MR; Shelley Medical 20 Imaging Technologies, Ontario, Canada) by attaching hoses with a $6 \mathrm{~mm}$ inner diameter to plastic 21 disks that attached the phantom to the PVC-box by compression. The pump was programmed to 22 simulate a carotid flow profile at a rate of 60 cycles $/$ min. A blood-mimicking solution of $40 \%$ 23 glycerin (Merck KGaA, Darmstadt, Germany) and 60\% deionized water mixture was pumped 
1 through the phantom at peak flow rates $10,20,30$, and $35 \mathrm{~mL} / \mathrm{s}$. The pump was purged before the

2 experiments to remove air bubbles from the solution.

\section{Data Aquisition}

Sonomicrometry data and ultrasound cineloops of the plaque and the vessel wall were collected at every flow rate, as seen in Fig. 3, for each of the four phantoms. Sonomicrometry data were collected for $30 \mathrm{~s}$ as a reference strain using a digital sonomicrometry (Sonometrics, Ontario,

Canada) acquisition system sampling at $200 \mathrm{~Hz}$. Sonomicrometry crystals $1 \mathrm{~mm}$ in diameter were super-glued (Loctite, Düsseldorf, Germany) to the plaque lumen and posterior phantom wall (Fig. 1b). The lumen crystal was placed on the cross-sectional apex of the bulging plaque such that it did not touch surrounding vessel walls while simultaneously positioned in-plane with the external crystals. Ultrasound image acquisition was turned off during sonomicrometry data collection to avoid interference between the systems.

Ultrasound long-axis B-mode images were collected on a Vivid E9 ultrasound machine (GE, Horten, Norway) with a fixed 9LD linear array transducer. Long-axis cineloops were recorded throughout 3 cycles for each flow rate at a frame rate of $42 \mathrm{~Hz}$, center frequency $10 \mathrm{MHz}, 3.5 \mathrm{~cm}$ depth, one focal point on each phantom wall, with image compounding turned off. The imaging plane was positioned close to the plane of the sonomicrometry crystals making them visible. Fig. 2b shows a sample B-mode long-axis image of the phantom with plaque inclusion embedded in the agar mixture. A schematic of the experimental setup is shown in Fig. 2c.

\section{Data Analysis}

The collected sonomicrometry and B-mode data were analyzed in Matlab 2011a (MathWorks, Natick, MA, USA) and synchronized by recording a simulated electrocardiogram (ECG) signal from the CompuFlow 1000MR pump. An in-house developed speckle tracking algorithm calculated 
1 the peak plaque strain which was compared to the strain found from the movement recorded by the

2 sonomicrometry crystals.

1) Speckle Tracking Analysis

The in-house speckle tracking algorithm (Larsson et al. 2011a) based on normalized crosscorrelation was used to perform the speckle tracking analysis. Axial (along the ultrasound beam) and lateral (orthogonal to the ultrasound beam) motion estimation was performed on 3 consecutive

7 pump cycles on both the radiofrequency signal as well as the envelope-detected signal with a kernel

8 length and width of 3 and 5 wavelengths $(\lambda)$ respectively. The kernel size was selected based on

9 pervious successful experiments measuring strain in the carotid wall both in-vitro (Larsson et al. 2011b) and in-vivo (Larsson et al. 2013). The kernel was moved in a search area which was defined

11 to allow maximum velocities of $2 \mathrm{~cm} / \mathrm{s}$ with a $60 \%$ axial and lateral overlap using spline

12 interpolation to detect sub-sample motion. Accumulation of the displacement maps throughout the cardiac cycle was then performed using linear interpolation to account for subpixel motion to assess

14 the cumulative strain. The radial (along the vessel radius) and longitudinal (along the vessel axis) speckle tracking strain curves were then obtained by linear regression in a region of interest (ROI) and subsequently low pass filtered with an averaging window 3 samples in length to remove noise.

17 All parameters described were chosen based on a previous study in a carotid artery phantom wall

18 (Larsson et al. 2011b) then modified to optimize the algorithm for measuring strain in plaques.

\section{a) Plaque Strain Estimation}

The ROI size was based on diastolic measurements of the size of the plaque. The ROI was manually selected near the vertical center of the plaque, offset to the right of the sonomicrometry crystal in the lumen to avoid effects of shadowing. The ROI for radial strain was selected to be 
1 approximately $50 \%$ of the distance from the crystal to the apex of the plaque (typically 4 mm width)

2 and $1.5 \mathrm{~mm}$ in height.

3 b) Vessel Wall Strain Estimation

4 Two of the phantoms were used for comparison between vessel wall and plaque strain. The 5 vessel wall ROI was placed on the same posterior side of the phantom as the plaque to achieve

6 similar boundary conditions from the PVC-box and agar-mixture. The ROI was manually placed 7 approximately $2 \mathrm{~cm}$ from the plaque in the center of the vessel wall with a ROI width and height of $8 \quad 1.5 \times 1.5 \mathrm{~mm}$ and $5 \times 1.5 \mathrm{~mm}$ in the radial and longitudinal directions respectively.

2) Sonomicrometry Analysis

10 Outliers from the inter-crystal displacement curves were removed by thresholding, followed by 11 filtering the resulting signals with a median filter 10 samples in length. Five cycles were averaged 12 to obtain the final reference displacement curves. Since the crystals were positioned in a triangle, 13 the law of cosines was used to calculate the radial displacement, whereas the longitudinal 14 displacement was the distance between crystal 2 and 3 (Fig. 1b). Thus, the strain $(\varepsilon(t))$ was 15 calculated as

$$
\varepsilon(\mathrm{t})=\frac{D(t)-D_{0}}{D_{0}}
$$

where $D_{0}$ is the initial distance between the crystals and $D(t)$ is the distance between the crystals at time $t$.

\section{E. Speckle Tracking Sonomicrometry Comparison}

The root mean square error (RMSE) was calculated on the first cycle of B-mode data for each

21 flow condition per phantom as

$$
\operatorname{RMSE}=\sqrt{\sum_{\mathrm{n}=1}^{\mathrm{N}} \frac{(\varepsilon(\mathrm{n})-\hat{\varepsilon}(\mathrm{n}))^{2}}{\mathrm{~N}}}
$$


1 where $\varepsilon(n)$ is the reference sonomicrometry strain, $\widehat{\varepsilon}(n)$ is the estimated speckle tracking strain,

2 and $\mathrm{N}$ is the number frames in one pump cycle. The absolute peak error was calculated as the

3 absolute difference between the reference and estimated peak systolic strain $\left(\left|\varepsilon_{\text {peak }}-\widehat{\varepsilon}_{\text {peak }}\right|\right)$ and

4 averaged for all flow conditions and phantoms. Bland-Altman plots for sonomicrometry and

5 speckle tracking peak strain were created and correlation analysis was performed. A paired t-test

6 was performed to compare the plaque and wall peak strain.

\section{IN VIVO EXPERIMENTS}

F. Data Aquisition

9 Long-axis and short-axis ultrasound cineloops were collected from 7 patients (mean age $77 \pm 6$

10 years, 4 male, 3 female) on a GE Vivid E9 ultrasound machine (Horten, Norway) with a handheld

11 9LD linear array transducer by an experienced ultrasound technician. The images were acquired

12 with a center frequency of $10 \mathrm{MHz}, 3.5 \mathrm{~cm}$ depth, two focal points on the arterial walls, with image

13 compounding turned off at a frame rate of $42 \mathrm{~Hz}$, similar to the in vitro setup. The procedure

14 consisted of collecting 3 long-axis and short-axis cineloops over 3 consecutive cardiac cycles for

15 the common carotid artery, the bulb, and the internal carotid artery. Patient medical data was

16 collected including sex, age, and blood pressure. The data were collected at Karolinska University

17 Hospital in Huddinge, Sweden with consent given by the patients and approval by the ethical

18 review board.

G. Data Analysis

1) Plaque Classification

21 Prior to speckle tracking analysis, the cineloops from the patients were visually analyzed and scored by two experienced physiologists. If a plaque was present and of sufficient size (diameter >

$232 \mathrm{~mm}$ ), the physicians outlined the borders in diastole, which later served as boundaries for ROI 
1 placement during speckle tracking analysis. The cineloops were scored using four different

2 measures. First, the plaque was classified homogeneous or heterogeneous. Homogeneous plaques

3 have a uniform texture and contain uniform echoes with medium to high level intensity compared

4 with the nearby adventitia tissue. Heterogeneous plaques have mixed level echoes and contain one

5 or more echolucent areas (low-level echoes). Plaque echogenicity was compared with echogenicity

6 of blood and adjacent adventitia. Low-level echoes were defined as those approaching echo levels

7 of blood, and medium- to high-level echoes were defined as echo strength similar or greater than

8 adventitia or adjacent soft tissue. Second, the plaque was classified if the surface was smooth or

9 irregular based on visual interpretation of greyscale and color Doppler images (Fitzgerald and

10 O'Farrell 1993; Kanber et al. 2013b).

11 The third method was to subjectively classify the plaque on a Gray-Weale 4-level scale; type 1:

12 mainly echolucent lesions; type 2: primarily echolucent lesions with some areas of echogenicity;

13 type 3: dominantly echogenic; type 4: uniformly echogenic. Types 1 and 2 correspond to

14 heterogeneous plaques and types 3 and 4 correspond to homogeneous plaques, which consist of 15 mainly fibrous tissue (Bluth 1997).

16 Fourth, the GSM of the plaque was measured using Automated Measurement System (AMS),

17 which was developed by the Department of Signals and Systems at Chalmers University of 18 Technology, Gothenburg, Sweden and the Physiology group at the Wallenberg laboratory, 19 Gothenburg University, Gothenburg, Sweden. The GSM was measured on a single frame of the 20 cineloop in systole and used as an alternative measurement of echogenicity. The image was 21 normalized by semi-automatically selecting the brightest and darkest pixel in the image followed by 22 calculating the GSM from image analyses of the physician-outlined plaque returning a median pixel 23 value in the range of 0 (black) to 255 (white).

24 2) Speckle Tracking Analysis 
After plaque scoring, the cineloops were analyzed with the in-house speckle tracking algorithm

2 with similar parameters as in the phantom setup. If the plaque diameter was $<2 \mathrm{~mm}$, the kernel size was reduced to a width and length of $2 \lambda$ and $4 \lambda$ respectively to allow for a smaller ROI. The ROI

$4 \quad$ varied with plaque size and shape and was made as large as possible without exceeding the outlined

5 plaque borders and manually placed in the center of the plaque as identified by the physiologists.

6 The speckle tracking strain curves were low pass filtered with an uniform averaging window 3

7 samples in length to remove noise. Plaque strain was estimated in all three carotid artery locations

8 given that plaque was present, of adequate size (diameter $>1.5 \mathrm{~mm}$ ), and that the image was of

9 sufficient quality. The peak negative radial and peak positive longitudinal strain in the plaque from

10 two cardiac cycles was calculated and averaged for each plaque. Thereafter, the radial and

11 longitudinal peak strain was adjusted for the patients' pulse-pressure (the difference in systolic and 12 diastolic blood pressure). Pulse-pressure adjusted peak negative radial and positive longitudinal 13 strain, GSM, and GSM variance (the variance of pixel values within the plaque) were compared 14 between plaques scored on the Gray-Weale scale.

\section{RESUlts}

\section{H. In vitro}

17 Sample speckle tracking and sonomicrometry plaque strain curves for radial and longitudinal strain can be seen in Fig. 4a and Fig. 4c respectively. Fig. 4 (b, d) shows the correlation plots of 19 speckle tracking versus sonomicrometry for radial $(\mathrm{r}=0.96, \mathrm{p}<0.001)$ and longitudinal $(\mathrm{r}=0.75$, $20 \mathrm{p} \leq 0.01)$ peak strain respectively. The radial and longitudinal Bland-Altman plots can be seen in 21 Fig. 5a and Fig. 5b respectively. Radial and longitudinal Limits of Agreement (LOA) were $-4.40 \%$ to $3.48 \%$ and $-2.29 \%$ to $2.78 \%$ respectively. Table 1 shows the mean and standard deviation (SD)

23 for the absolute peak error and RMSE for the radial and longitudinal strain. Longitudinal strain data 24 was discarded for one of the phantoms due to irregular movement caused by fluid in the plaque 
1 cavity. The plaque exhibited $35.1 \pm 16.9 \%$ greater radial $(\mathrm{p}<0.001)$ and $88.6 \pm 72.0 \%$ greater

2 longitudinal $(\mathrm{p}<0.001)$ peak strain compared to the arterial wall when measured with speckle

3 tracking.

4 I. In vivo

5 Sixteen plaques were present in the patient population of which 7 data points were removed due

6 to poor image quality or too small plaque size $(n=9)$. Fig. 6 shows the pulse-pressure adjusted mean

7 of peak strains and standard deviations for plaques in the radial (a) and longitudinal (b) directions

8 sorted by Gray-Weale classification. The mean of the peak strain values and standard deviations for

9 radial and longitudinal plaque strain as well as the pulse-pressure adjusted values can be seen in

10 Table 2 sorted by Gray-Weale classification.

11 Table 3 shows the plaques locations and classification results. A sample echogenic and

12 echolucent plaque can be seen in Fig. 7a and Fig. 7d respectively with a physician outlined border

13 (green line) and ROI (red rectangle). Fig. 7 also shows in vivo sample speckle tracking strain curves

14 in the radial (b, e) and longitudinal (c, f) directions for the echogenic and echolucent plaques

15 respectively.

16 The measured mean and standard deviation plaque GSM by Gray-Weale classification was: type

171 GSM $40 \pm 0$; type 2 GSM $84 \pm 9$; and type 3 GSM 53. Fig. 8 shows GSM variance in the plaque 18 versus Gray-Weale classification.

\section{Discussion}

20 Noninvasive characterization of carotid artery plaque based on quantitative data would be a 21 valuable tool for physicians to determine treatment options for patients with atherosclerosis. Current 22 standards limit physicians to visual assessment of ultrasound B-mode images and carotid 23 angiograms allowing observer error to influence the results (Arnold et al. 1999). The goal of this 
1 study was to validate an in-house speckle tracking algorithm based on block matching to quantify

2 the strain of the plaque in a phantom setup with sonomicrometry and to perform a small pilot study

3 in vivo. To the best of our knowledge assessment of plaque strain in ultrasound carotid images has

4 not been validated via an independent method.

5 J. In vitro Study

6 The validation study indicates good agreement between the speckle tracking algorithm and the 7 sonomicrometry. A strong correlation between speckle tracking and reference strain values was

8 found radially (Fig. 4b), while the agreement in the longitudinal direction (Fig. 4d) was not as

9 strong but still good. This was most likely due to the intrinsic lower resolution in the lateral

10 direction coupled with little longitudinal plaque movement. The radial and longitudinal absolute

11 peak error and RMSE (Table 1) indicates less absolute error in the longitudinal strain. However, it

12 must be taken into consideration that the radial strain is significantly larger in magnitude than the

13 longitudinal strain. The radial and longitudinal Bland-Altman plots (Fig. 5) show that the speckle

14 tracking algorithm overestimates low strain values and underestimates high strain values. Radially,

15 the LOA are acceptable in relation to the range whereas the longitudinal LOA are higher as a result

16 of two outliers (Fig. 5b). Nevertheless, there is still good correlation $(\mathrm{r}=0.75)$ between

17 sonomicrometry and speckle tracking in the longitudinal direction.

18 Creating a phantom with a soft plaque inclusion was challenging resulting in several design

19 modifications. Eight phantoms were attempted of which only four succeeded. The first two

20 successful phantoms leaked fluid into the plaque cavity when connected to the pump. This resulted

21 in less radial and longitudinal plaque strain combined with longitudinal plaque compression in one

22 of the phantoms. Furthermore, the fluid degraded the image quality of the plaque. The final two

23 phantoms were modified by super-gluing the plaque cavity shut after the final freeze cycle to 
1 prevent fluid from the lumen to leak into the cavity. These phantoms were used for the strain

2 comparison between plaque and vessel wall.

3 A second source of error was the placement of the lumen sonomicrometry crystal on the plaque

4 (Fig. 1b crystal 1). Limited visibility and accessibility made it difficult to place the crystal on the

5 center of the lumen plaque extrusion. Off-center placement can result in underestimation of radial

6 displacement. The fairness of comparing sonomicrometry and speckle tracking could also be

7 questioned since the crystals and ROIs were not placed at the exact same location in the plaque to

8 avoid the shadowed region under the crystals (Fig. 2b).

9 During the cardiac cycle, the radial strain in the phantom plaque had a significant gradient as a

10 result of greater plaque movement near the lumen in contrast to the plaque boundary facing the

11 posterior wall. To avoid these extremes, the ROI was placed in the center of the plaque in

12 combination with limiting the ROI height to $50 \%$ of the vertical plaque size in order to measure an

13 average value for the plaque strain. The strain gradient was not significant in the in vivo experiment

14 as it is an effect due to the experimental setup from the boundary conditions imposed on the

15 phantom by the agar mixture and glycerin blood mimicking fluid. It should be mentioned that a

16 moving the kernel in a search defined to allow maximum velocities of $2 \mathrm{~cm} / \mathrm{s}$ can be a potential

17 tracking limitation of the speckle tracking algorithm; however, we did not experience this limitation

18 in-vivo or in-vitro. Additionally, the algorithm does not take into account out-of-plane motion. In

19 other strain studies involving carotid artery mimicking phantoms, the vessel was placed in a water

20 bath while the intraluminal pressure was varied statically (Ribbers et al. 2007). Initial pilot tests

21 indicated that the flow profiles from the pump induced a ringing artifact in the phantom at the end

22 of the cardiac cycle when surrounded by water in the fixture. By mimicking the boundary

23 conditions imposed by the surrounding tissue with the agar-mixture the ringing was damped and the

24 axial motion in the phantom was similar to that of the programed flow profile. It should also be 
1 noted that in this experimental setup a plaque in the common carotid artery was mimicked, but it is

2 more common for plaque buildup to occur in the artery bifurcation (Oviedo et al. 2010).

3 Despite these limitations, the two methods are in good agreement and it is possible to distinguish

4 the plaque from the arterial wall. These results are in line with other studies showing that it is

5 feasible to measure plaque strain in vivo (de Korte et al. 2002; Golemati et al. 2012; Le Floc'h et al.

6 2012; McCormick et al. 2012; Schmitt et al. 2007). In addition, Schaar (Schaar et al. 2003) found a

7 high sensitivity (88\%) and specificity (89\%) when characterizing plaque with strain using IVUS.

8 Other techniques have used accumulated axial strain and relative lateral shifts as intricacies for

9 plaque strains using IVUS with positive results (Shi et al. 2008a), yet these techniques are invasive.

\section{$10 \quad$ K. In vivo Study}

11 The study shows that it is possible to estimate plaque strain via speckle tracking in vivo using B-

12 mode ultrasound. The results show an increase in radial strain for mainly echolucent plaques in

13 contrast to dominantly echogenic plaques (Fig. 6a). This is in agreement with our hypothesis that

14 the force from the blood of the cardiac systolic pressure wave induces greater deformation in

15 echolucent plaques with a large lipid or intraplaque hemorrhage core in contrast to echogenic

16 plaques composed of relatively homogeneous fibrous tissue. Gray-Weale type 1 and 2 plaques

17 exhibited increased longitudinal strain compared to the type 3 plaque (Fig. 6b). Both radial and

18 longitudinal strain results were normalized to the patient's pulse pressure since it is the driving

19 force for radial strain and one of the forces, in addition to shear forces from blood flow and 20 tethering forces from the heart, for longitudinal strain. Without normalization, variation in blood 21 pressure between patients could bias the results.

22 Sample strain curves show increased radial (Fig. 7b, e) and longitudinal strain (Fig. 7c, f) for an 23 echolucent plaque (Fig. 7d) compared to an echogenic plaque (Fig. 7a). The strain curves also 24 indicate the reproducibility over two cardiac cycles given that the ultrasound cineloop is of good 
1 quality. Reproducibility requires the transducer and patient to be motionless for approximately $3 \mathrm{~s}$

2 during image acquisition. In practice, this is quite difficult for elderly patients, especially those with

3 additional ailments and illnesses. Given the small arterial motions and strains, stabilization of the

4 ultrasound probe in a fixture would be essential in clinical applications and could potential improve

5 results and reproducibility. Furthermore, the plaque must be of sufficient size (diameter $>2 \mathrm{~mm}$ ) in

6 order to achieve reliable measurements. These two constraints limited us from successfully

7 measuring strain in all in vivo plaque data. The in-vivo waveforms (Fig. 7b, c, e, f) vary more from

8 cycle to cycle compare to the in-vitro waveforms in Fig. 4a, c. This can be explained by the fact that

9 in-vitro phantoms are connected to a pump controlled by a precision stepper motor, while the in-

10 vivo arterial wall motion is driven by a variable heart rate and stroke volume. The in-vivo

11 longitudinal strains (Fig. 7c, f) are also influence by different shear forces than the in-vitro phantom

12 vessel due to more complex arterial wall and plaque surface composition as well as differences in

13 fluid properties between blood and the blood-mimicking glycerin mixture used in the phantom,

14 which also could attribute to the more complex in-vivo strain waveforms. Moreover, the in-vitro

15 longitudinal strain does not experience any of the tethering forces from the pumping of the heart,

16 which influences the longitudinal in-vivo strain.

17 de Korte et al. (2002) investigated the identification of atherosclerotic plaque components in 18 external iliac and femoral arteries using IVUS elastography in an in vivo Yucatan pig study. Peak radial strain values up to $2 \%$ for echolucent plaques were recorded, which is slightly less than our 20 absolute peak radial strain values (4-7\%) for echolucent plaques. Different arterial locations and pressures, as well as different species may explain the difference in measured strain.

Sztajzel et al. (2005) and Salem et al. (2012) found that plaques with large calcifications 23 presented the highest GSM values, fibrous plaques had intermediate GSM values, and those with 24 large hemorrhagic areas or with a predominant necrotic core exhibited the lowest. Nevertheless, in 
1 our limited data set no relationship was found between GSM and plaque echogenicity, which could

2 be attributed to measuring the GSM on a single frame rather than averaging the GSM throughout

3 the cardiac cycle. However, Fig. 8 shows that GSM variance was greater for echolucent plaques

4 compared to the echogenic plaque. The reliability of GSM as a quantitative tool for measuring

5 plaque vulnerability has been disputed. GSM is an overall measure of plaque echogenicity, making

6 it possible for a plaque to have average GSM values even with highly echolucent areas due to

7 counterbalancing echogenic areas. It has recently been shown that there is no additional value of the

8 use of GSM in evaluating plaque vulnerability (Kolkert et al. 2014), which is in contrast to previous

9 studies (Sabetai et al. 2000).

10 Some study limitations deserve to be pointed out. Generally, classifying atherosclerotic plaques

11 as "hard" or "soft" is most likely an oversimplification. From our small sample population we found

12 that the majority of plaques are a mixture of echogenic and echolucent regions making them very

13 difficult to classify on the Gray-Weale scale, allowing for subjectivity in the interpretation. In

14 addition, it is very difficult to classify plaques strictly on long-axis B-mode images, as clinicians are

15 accustomed to using long-axis and short-axis images, as well as Doppler color flow (Arnold et al.

16 1999). Certain plaque-lumen borders were not clearly defined making it difficult for the physicians

17 to outline the plaque, as well as assess the plaque surface. Qualitative assessment of plaque surface

18 irregularities is subjective, but more quantitative methods are being developed (Kanber et al.

19 2013b). A larger and more variable sample population would have been desirable with several

20 plaques in all Gray-Weale scale categories. We acknowledge that the study is limited due to the

21 presence of only one type 3 dominantly echogenic plaque. However, we emphasize that these are

22 preliminary results and we plan to conduct a larger clinical study in the future.

23 Measuring strain in plaques with a mixture of echolucent and echogenic regions can be difficult.

24 In complex plaque geometries with mixed regions, such as Fig. 7a, it is not intuitive where to place 
1 the rectangular ROI. We attempted to place the ROI in both echolucent and echogenic regions of

2 the plaque to get an average strain value for the motion in both regions.

3 Alternative motion tracking methods could potentially improve plaque tracking in future studies.

4 Golemati et al. (2012) performed a comparison of block matching and differential methods for

5 motion analysis of the carotid artery wall. Four techniques were compared: optical flow; weighted

6 least-squares optical flow; block matching; and affine block motion model. It was found that the

7 least-squares optical flow performed best on simulated B-mode images. However, the study lacked

8 validation against an independent strain measurement method, such as sonomicrometry.

9 Future in vivo studies should include a larger sample population and a ROI that follows the

10 physician plaque outline. A contrast agent could potentially improve the edge detection of the

11 plaques resulting in improved motion tracking. To avoid subjective plaque classification, the sample

12 population should consist of patients undergoing an endarterectomy procedure to allow for plaque

13 classification via histology. If a large enough plaque strain database is collected and validated

14 against histology, physicians could potentially use the technique to assess the vulnerability of

15 plaques. Finally, this study did not include circumferential plaque strain, which could be of 16 potential clinical significance.

\section{CONCLUSION}

18 This study shows the feasibility of radial and longitudinal speckle tracking strain estimation of 19 plaque in the carotid artery which was validated by sonomicrometry in a plaque phantom. The 20 results indicate the possibility of characterizing plaque vulnerability based on strain, but the 21 algorithm must be applied in a larger study to build a plaque strain database validated against 22 histology. Preliminary results show increased radial and longitudinal strain in dominantly 23 echolucent and primarily echolucent plaques with some areas of echogenicity, compared with strain 24 in echogenic plaques. 


\section{ACKNOWLEDGEMENTS}

2 This study was supported by VINNOVA VINNMER Marie Curie International qualification, the 3 Swedish Heart-Lung Foundation, and the Swedish Research Council. We would like to thank Rita

4 Balzano and Linnea Sandersjö for collecting in vivo ultrasound images and Dr. Kent Lund for 5 performing the clinical plaque classification. 
2 Allen JD, Ham KL, Dumont DM, Sileshi B, Trahey GE, Dahl JJ. The development and potential of 3 acoustic radiation force impulse (ARFI) imaging for carotid artery plaque characterization. Vasc Med 2011;16:302-11.

Armas R, Kirkwood JR, Zubi S. The posterior cerebral radionuclide angiogram for the detection of carotid artery disease. Clin Nucl Med 1981;6:19-22.

Arnold JA, Modaresi KB, Thomas N, Taylor PR, Padayachee TS. Carotid plaque characterization by duplex scanning: observer error may undermine current clinical trials. Stroke 1999;30:615.

Biasi GM, Froio A, Diethrich EB, Deleo G, Galimberti S, Mingazzini P, Nicolaides AN, Griffin M, Raithel D, Reid DB, Valsecchi MG. Carotid plaque echolucency increases the risk of stroke in carotid stenting: the Imaging in Carotid Angioplasty and Risk of Stroke (ICAROS) study. Circulation 2004;110:756-62.

Biasi GM, Sampaolo A, Mingazzini P, De Amicis P, El-Barghouty N, Nicolaides AN. Computer analysis of ultrasonic plaque echolucency in identifying high risk carotid bifurcation lesions. Eur J Vasc Endovasc 1999;17:476-9.

Bluth EI. Evaluation and characterization of carotid plaque. Seminars in ultrasound, CT, and MR 1997;18:57-65.

Dahl JJ, Dumont DM, Allen JD, Miller EM, Trahey GE. Acoustic radiation force impulse imaging for noninvasive characterization of carotid artery atherosclerotic plaques: a feasibility study. Ultrasound Med Biol 2009;35:707-16.

de Korte CL, Sierevogel MJ, Mastik F, Strijder C, Schaar JA, Velema E, Pasterkamp G, Serruys $\mathrm{PW}$, van der Steen AF. Identification of atherosclerotic plaque components with intravascular ultrasound elastography in vivo: a Yucatan pig study. Circulation 2002;105:1627-30. 
1 Deyama J, Nakamura T, Takishima I, Fujioka D, Kawabata K, Obata JE, Watanabe K, Watanabe Y,

2 Saito Y, Mishina H, Kugiyama K. Contrast-enhanced ultrasound imaging of carotid plaque neovascularization is useful for identifying high-risk patients with coronary artery disease. Circ J 2013;77:1499-507.

5 Easton JD, Wilterdink JL. Carotid endarterectomy: trials and tribulations. Annals of neurology 1994;35:5-17.

7 Fitzgerald DE, O'Farrell CM. Prognostic value of ultrasound morphology in carotid atherosclerosis. International angiology : a journal of the International Union of Angiology 1993;12:337-41. author reply e8.

Golemati S, Stoitsis JS, Gastounioti A, Dimopoulos AC, Koropouli V, Nikita KS. Comparison of ultrasound images. IEEE Trans Inf Technol Biomed 2012;16:852-8.

Gray-Weale AC, Graham JC, Burnett JR, Byrne K, Lusby RJ. Carotid artery atheroma: comparison of preoperative B-mode ultrasound appearance with carotid endarterectomy specimen pathology. J Cardiovasc Surg (Torino) 1988;29:676-81.

Kakkos SK, Griffin MB, Nicolaides AN, Kyriacou E, Sabetai MM, Tegos T, Makris GC, Thomas DJ, Geroulakos G, Ri ACS. The size of juxtaluminal hypoechoic area in ultrasound images of asymptomatic carotid plaques predicts the occurrence of stroke. J Vasc Surg 2013;57:609-17.

Kanber B, Hartshorne TC, Horsfield MA, Naylor AR, Robinson TG, Ramnarine KV. Dynamic variations in the ultrasound greyscale median of carotid artery plaques. Cardiovasc Ultrasound 2013a;11:21. 
1 Kanber B, Hartshorne TC, Horsfield MA, Naylor AR, Robinson TG, Ramnarine KV. Quantitative assessment of carotid plaque surface irregularities and correlation to cerebrovascular symptoms. In: ed. Cardiovasc Ultrasound. England: 2013b. pp. 38.

Kolkert JL, Meerwaldt R, Loonstra J, Schenk M, van der Palen J, van den Dungen JJ, Zeebregts CJ.

Relation between B-mode gray-scale median and clinical features of carotid stenosis vulnerability. Annals of vascular surgery 2014;28:404-10.

Larsson M, Kremer F, Claus P, Kuznetsova T, Brodin LA, D'hooge J. Ultrasound-Based Radial and Longitudinal Strain Estimation of the Carotid Artery: A Feasibility Study. IEEE Trans Ultrason Ferroelectr Freq Control 2011a;58:2244-51.

Larsson M, Kremer F, Heyde B, Brodin LA, D'hooge J. Ultrasound-based Speckle Tracking for 3D Strain estimation of the Arterial wall - An experimental validation study in a tissue mimicking phantom. IEEE International Ultrasonics Symposium 2011b;725-8.

Larsson M, Verbrugghe P, Smoljkic M, Heyde B, Famaey N, Herijgers P, D'Hooge J. Assessment of longitudinal strain in the carotid artery wall using ultrasound-based Speckle tracking Validation in a sheep model. IEEE International Ultrasonics Symposium 2013;569-72.

Le Floc'h S, Cloutier G, Saijo Y, Finet G, Yazdani SK, Deleaval F, Rioufol G, Pettigrew RI, Ohayon J. A four-criterion selection procedure for atherosclerotic plaque elasticity reconstruction based on in vivo coronary intravascular ultrasound radial strain sequences. Ultrasound Med Biol 2012;38:2084-97.

Mahmoud AM, Dutta D, Lavery L, Stephens DN, Villanueva FS, Kim K. Noninvasive detection of lipids in atherosclerotic plaque using ultrasound thermal strain imaging: in vivo animal study. J Am Coll Cardiol 2013; 
1 Matos JM, Barshes NR, McCoy S, Pisimisis G, Felkai D, Kougias P, Lin PH, Bechara CF.

2 Validating common carotid stenosis by duplex ultrasound with carotid angiogram or

3 computed tomography scan. J Vasc Surg 2014;59:435-9.

4 McCormick M, Varghese T, Wang X, Mitchell C, Kliewer MA, Dempsey RJ. Methods for robust in $5 \quad$ vivo strain estimation in the carotid artery. Phys Med Biol 2012;57:7329-53.

6 Mono ML, Karameshev A, Slotboom J, Remonda L, Galimanis A, Jung S, Findling O, De Marchis

7 GM, Luedi R, Kiefer C, Stuker C, Mattle HP, Schroth G, Arnold M, Nedeltchev K, El-

Oviedo C, Maehara A, Mintz GS, Araki H, Choi SY, Tsujita K, Kubo T, Doi H, Templin B, Lansky Koussy M. Plaque characteristics of asymptomatic carotid stenosis and risk of stroke. Cerebrovasc Dis 2012;34:343-50. AJ, Dangas G, Leon MB, Mehran R, Tahk SJ, Stone GW, Ochiai M, Moses JW. Intravascular ultrasound classification of plaque distribution in left main coronary artery bifurcations: where is the plaque really located? Circ Cardiovasc Interv 2010;3:105-12.

Prahl U, Holdfeldt P, Bergstrom G, Fagerberg B, Hulthe J, Gustavsson T. Percentage white: a new feature for ultrasound classification of plaque echogenicity in carotid artery atherosclerosis. Ultrasound Med Biol 2010;36:218-26.

Ramnarine KV, Garrard JW, Dexter K, Nduwayo S, Panerai RB, Robinson TG. Shear wave elastography assessment of carotid plaque stiffness: in vitro reproducibility study. Ultrasound Med Biol 2014;40:200-9.

Ramnarine KV, Hartshorne T, Sensier Y, Naylor M, Walker J, Naylor AR, Panerai RB, Evans DH. Tissue Doppler imaging of carotid plaque wall motion: a pilot study. Cardiovasc Ultrasound $2003 ; 1: 17$

Reckless IP, Buchan AM. Stroke: management and prevention. Medicine 2008;36:592-600. 
1 Reid DB. Carotid plaque characterization: helpful to endarterectomy and endovascular surgeons. J $2 \quad$ Endovasc Surg 1998;5:247-50.

3 Reiter M, Bucek RA, Effenberger I, Boltuch J, Lang W, Ahmadi R, Minar E, Schillinger M. Plaque $4 \quad$ echolucency is not associated with the risk of stroke in carotid stenting. Stroke 2006;37:2378-

5

6 Ribbers H, Lopata RGP, Holewijn S, Pasterkamp G, Blankensteijn JD, De Korte CL. Noninvasive 7 two-dimensional strain imaging of arteries: Validation in phantoms and preliminary experience in carotid arteries in vivo. Ultrasound Med Biol 2007;33:530-40.

Ronen RR, Clarke SE, Hammond RR, Rutt BK. Carotid plaque classification: defining the certainty with which plaque components can be differentiated. Magn Reson Med 2007;57:874-80.

Saba L, Argiolas GM, Siotto P, Piga M. Carotid artery plaque characterization using CT multienergy imaging. AJNR Am J Neuroradiol 2013;34:855-9.

Sabetai MM, Tegos TJ, Nicolaides AN, El-Atrozy TS, Dhanjil S, Griffin M, Belcaro G, Geroulakos G. Hemispheric symptoms and carotid plaque echomorphology. In: ed. J Vasc Surg. United States: 2000. pp. 39-49.

Salem MK, Sayers RD, Bown MJ, West K, Moore D, Nicolaides A, Robinson TG, Naylor AR. Patients with recurrent ischaemic events from carotid artery disease have a large lipid core and low GSM. Eur J Vasc Endovasc Surg 2012;43:147-53.

Schaar JA, De Korte CL, Mastik F, Strijder C, Pasterkamp G, Boersma E, Serruys PW, Van Der Steen AF. Characterizing vulnerable plaque features with intravascular elastography. Circulation 2003;108:2636-41.

Schmitt C, Soulez G Fau - Maurice RL, Maurice R1 Fau - Giroux M-F, Giroux Mf Fau - Cloutier G, Cloutier G. Noninvasive vascular elastography: toward a complementary characterization tool of atherosclerosis in carotid arteries. Ultrasound Med Biol 2007;33:1841-58. 
1 Shi H, Mitchell CC, McCormick M, Kliewer MA, Dempsey RJ, Varghese T. Preliminary in vivo

2 atherosclerotic carotid plaque characterization using the accumulated axial strain and relative 3 lateral shift strain indices. Phys Med Biol 2008a;53:6377-94.

4 Shi H, Varghese T, Dempsey RJ, Salamat MS, Zagzebski JA. Relationship between ultrasonic attenuation, size and axial strain parameters for ex vivo atherosclerotic carotid plaque.

$6 \quad$ Ultrasound Med Biol 2008b;34:1666-77.

7 Sztajzel R, Momjian S, Momjian-Mayor I, Murith N, Djebaili K, Boissard G, Comelli M, Pizolatto study. Eur J Radiol 2013; 


\section{Figure Captions}

2 Fig. 1. a) Acrylic block mold with bronze rod with extension to create plaque cavity. b) Long-axis

7 Fig. 2.a) Photo of the vessel phantom attached to the polyvinyl chloride (PVC) box connected to the

8 pump prior to filling the cavity with the agar-mixture. The wires are leads to the sonomicrometry

9 crystals. b) B-mode long-axis image of the phantom vessel with a soft plaque inclusion to the left in cross-sectional schematic illustration of the phantom with dimensions and placement of sonomicrometry crystals (1-3). Vessel dimensions: $100 \mathrm{~mm}$ in length; $6 \mathrm{~mm}$ lumen diameter; and $3 \mathrm{~mm}$ wall thickness. the posterior wall. The red box indicates the region of interest for the speckle tracking radial strain analysis offset to the right of the lumen sono-crystal to avoid the shadowed region. c) Diagram of the experimental setup illustrating connections between the phantom, sonomicrometry system, programmable pump, and ultrasound machine.

Fig. 3. CompuFlow 1000MR programmable pump carotid artery flow profile curves for 10, 20, 30, and $35 \mathrm{~mL} / \mathrm{s}$.

Fig. 4. Sample in vitro plaque strain curves for sonomicrometry (solid line) and speckle tracking (blue dashed line) for radial (a) and longitudinal (c) directions. Correlation plots for negative radial (a) and positive longitudinal (d) peak speckle tracking strain versus peak sonomicrometry strain along with correlation values.

Fig. 5. Radial (a) and longitudinal (b) Bland-Altman plots showing the difference between the sonomicrometry and speckle tracking strain versus the sonomicrometry strain. 
2 Fig. 6. Pulse-pressure (PP) adjusted means and standard deviations for radial (a) and longitudinal 3 (b) strains for in vivo plaques sorted by Gray-Weale classification.

4 Fig. 7 In vivo B-mode ultrasound images for an (a) echogenic plaque located in the right internal 5 carotid artery of a 72 year-old woman and an (d) echolucent plaque located in the left carotid artery

6 bulb of a 78 year-old man. The green line is the physicians' outline of the plaques and the regions of 7 interest for speckle tracking analysis are shown in red. Strain curves (black) for radial (b, e) and 8 longitudinal (c, f) directions for echogenic (left column) and echolucent (right column) plaques 9 respectively. The patient's ECG curves are displayed as blue dotted curves in figures b, c, e, and f. 10

11 Fig. 8. Gray Scale Median (GSM) variance versus Gray-Weale classification for in vivo plaques. 

STRAIN (ABSOLUTE PEAK ERROR) AS WELL AS THE RMSE.

\begin{tabular}{|lcc|}
\hline & $\begin{array}{c}\text { Radial strain } \\
\text { Mean } \pm \text { SD }\end{array}$ & $\begin{array}{c}\text { Longitudinal strain } \\
\text { Mean } \pm \text { SD }\end{array}$ \\
\hline Absolute Peak Error (\%) & $2.09 \pm 2.03$ & $1.61 \pm 1.57$ \\
RMSE (\%) & $2.93 \pm 2.18$ & $0.78 \pm 0.49$ \\
\hline
\end{tabular}

TABLE 2.

IN VIVO STRAIN ESTIMATION RESULTS FROM SPECKLE TRACKING ANALYSIS IN THE PLAQUES. MEAN OF PEAK STRAINS AND STANDARD DEVIATIONS IN THE RADIAL AND LONGITUDINAL DIRECTION AS WELL AS PULSE-PRESSURE (PP) ADJUSTED STRAIN SORTED BY GRAY-WEALE CLASSIFICATION.

\begin{tabular}{|ccccc|}
\hline $\begin{array}{c}\text { Gray-Weale } \\
\text { Classification }\end{array}$ & $\begin{array}{c}\text { Radial Strain } \\
\text { (\%) }\end{array}$ & $\begin{array}{c}\text { PP Adjusted Radial } \\
\text { Strain } \\
\left(\boldsymbol{x} \mathbf{1 0}^{-2} \mathbf{m m ~ H o ~}^{-1}\right)\end{array}$ & $\begin{array}{c}\text { Longitudinal Strain } \\
(\%)\end{array}$ & $\begin{array}{c}\text { PP Adjusted } \\
\text { Longitudinal Strain }_{\left(\mathbf{x 1 0}^{-2} \mathbf{m m H g}^{-1}\right)}\end{array}$ \\
\hline 1 & $-5.4 \pm 1.5$ & $-9.9 \pm 2.7$ & $1.3 \pm 0.7$ & $2.4 \pm 1.2$ \\
2 & $-2.7 \pm 1.4$ & $-5.0 \pm 2.9$ & $1.3 \pm 0.5$ & $2.1 \pm 1.0$ \\
3 & -2.6 & -2.9 & 0.7 & 0.8 \\
\hline
\end{tabular}

11 
1 TABLE 3. IN VIVO PLAQUE LOCATION AND CLASSIFICATION RESULTS. HOMOGENEOUS (HOMO)

2 VERSUS HETEROGENEOUS (HETERO) CONSISTENCY, SMOOTH (S) VERSUS ROUGH (R) SURFACE, VISUAL

3 CLASSIFICATION; ECHOLUCENT (L), DOMINANT ECHOLUCENT (DL), DOMINANT ECHOGENIC (DG), 4 EChOGENiC (G), AND GRAY-WEALE SCORE. ThE PlaQUES WERE LOCATED IN THE BULB, COMMON 5 CAROTID ARTERY (CCA), AND INTERNAL CAROTID ARTERY (ICA).

\begin{tabular}{|cccccc|}
\hline $\begin{array}{c}\text { Plaque } \\
\text { Number }\end{array}$ & Location* & $\begin{array}{c}\text { Homogeneous vs. } \\
\text { Heterogeneous }\end{array}$ & $\begin{array}{c}\text { Smooth vs. } \\
\text { Rough } \\
\text { surface }\end{array}$ & $\begin{array}{c}\text { Visual } \\
\text { Classification }\end{array}$ & $\begin{array}{c}\text { Gray-Weale } \\
\text { Score (1-4) }\end{array}$ \\
\hline 1 & Bulb & Hetero & $\mathrm{R}$ & $\mathrm{DL}$ & 2 \\
2 & ICA & Hetero & $\mathrm{R}$ & $\mathrm{DL}$ & 2 \\
3 & CCA & Hetero & $\mathrm{S}$ & $\mathrm{DL}$ & 2 \\
4 & ICA & Hetero & $\mathrm{R}$ & $\mathrm{DL}$ & 2 \\
5 & CCA & Hetero & $\mathrm{R}$ & $\mathrm{DL}$ & 2 \\
6 & Bulb & Homo & $\mathrm{R}$ & $\mathrm{DL}$ & 2 \\
7 & Bulb & Hetero & $\mathrm{R}$ & $\mathrm{DG}$ & 3 \\
8 & Bulb & Homo & $\mathrm{S}$ & $\mathrm{L}$ & 1 \\
9 & ICA & Homo & S & L & 1 \\
\hline
\end{tabular}

6

* Bulb $=$ Carotid Artery Bulb, CCA = Common Carotid Artery, ICA = Internal Carotid Artery

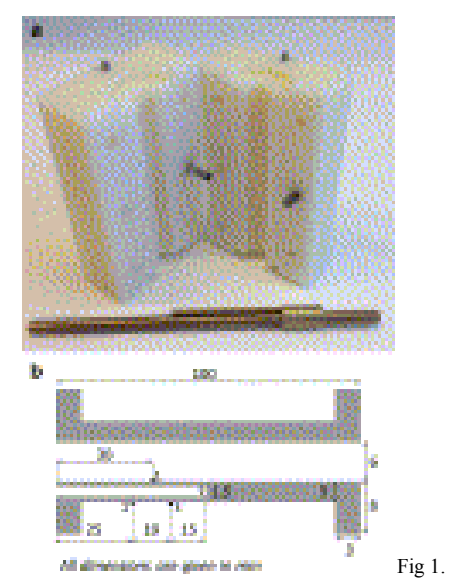



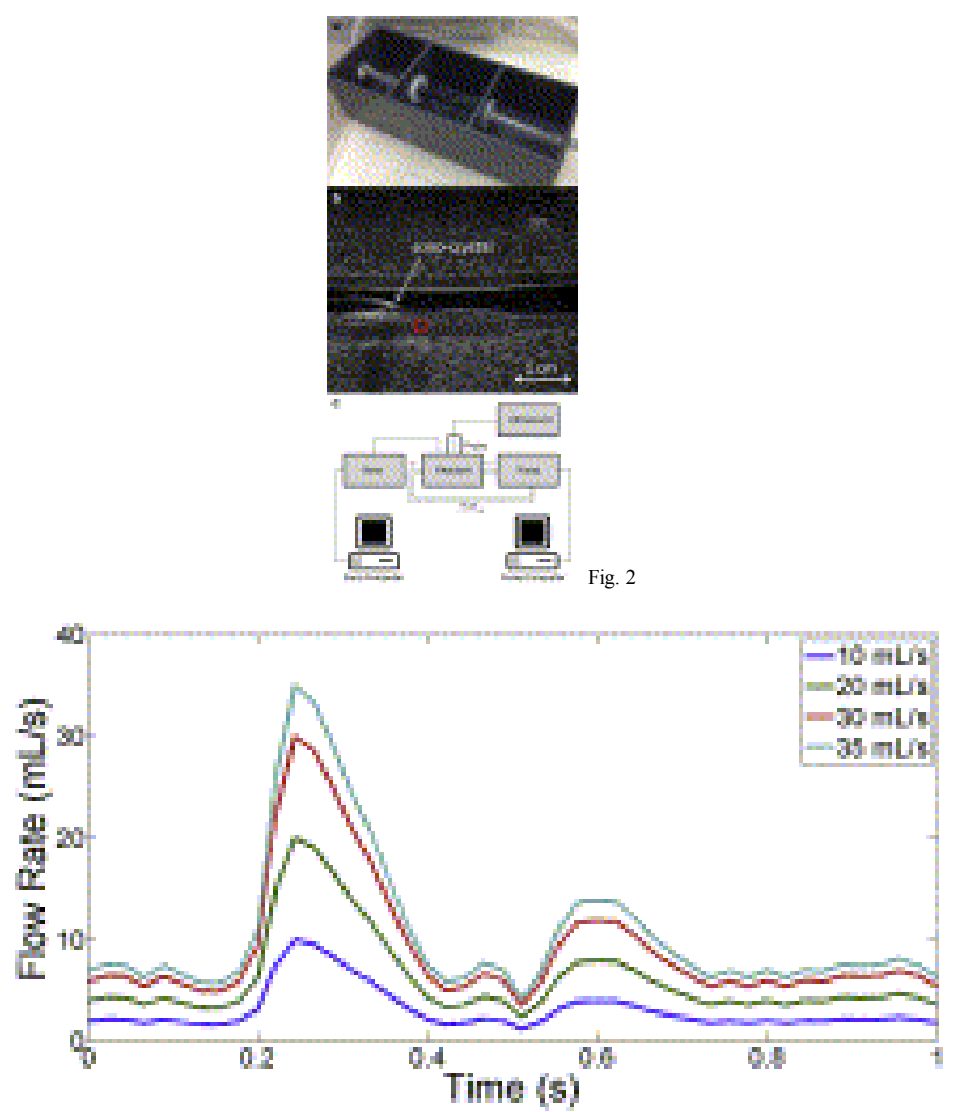

Fig. 3
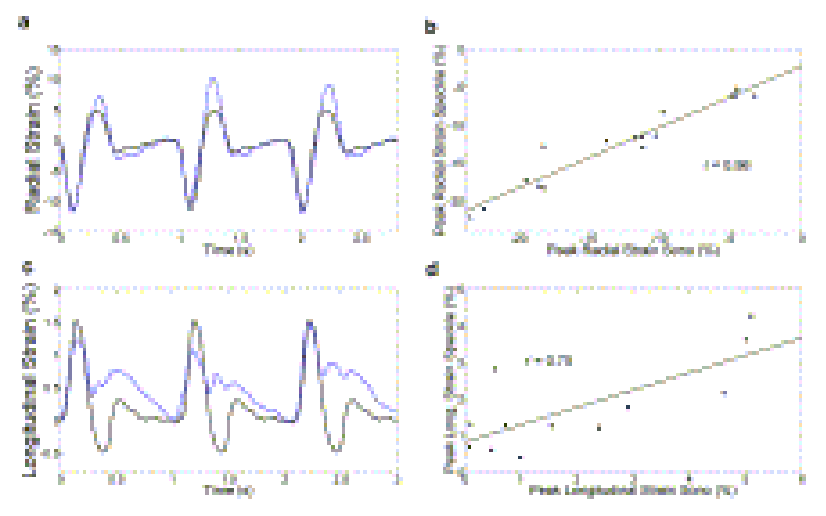

Fig 4

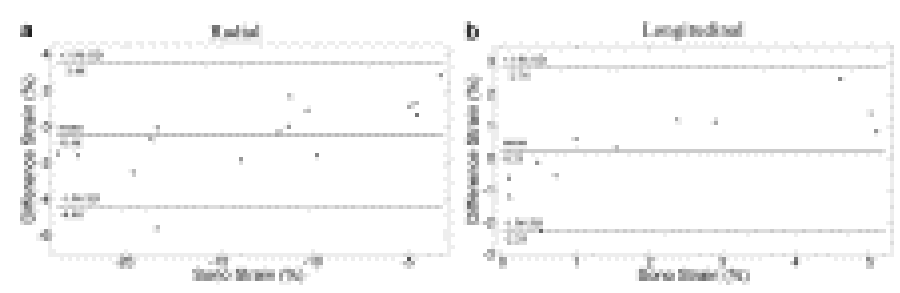

7

8 

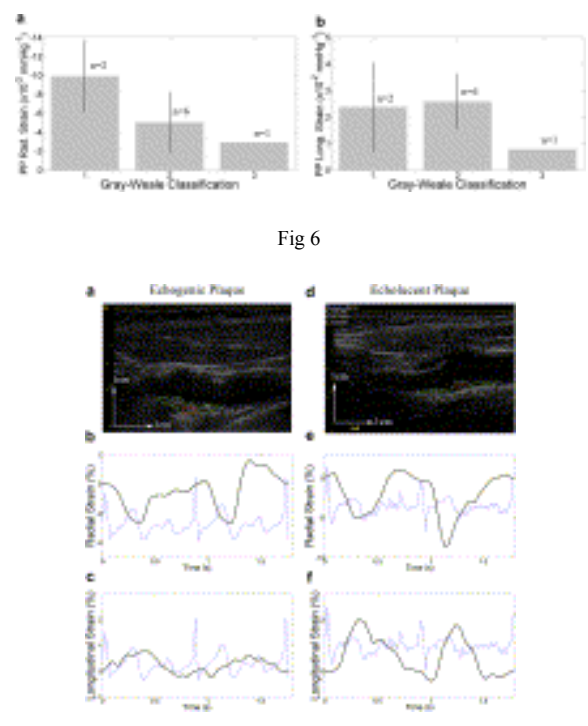

4

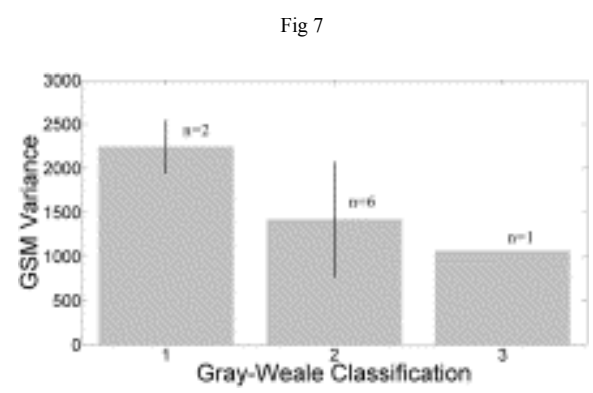

